\title{
Depth Measurement Bias in Pulsed Airborne Laser Hydrography Induced by Chromatic Dispersion
}

Type: Peer reviewed journal article.

Status: accepted June 12, 2020, published June 30, 2020

Version: Manuscript accepted for publication.

Copyright: IEEE subject to copyright policy for accepted versions of articles.

Bibliography: Roland Schwarz et al. "Depth Measurement Bias in Pulsed Airborne Laser Hydrography Induced by Chromatic Dispersion." In: IEEE Geoscience and Remote Sensing Letters (2020), pp. 1-5. DOI: 10.1109/lgrs.2020.3003088 



\title{
Depth Measurement Bias in Pulsed Airborne Laser Hydrography Induced by Chromatic Dispersion
}

\author{
Roland Schwarz, Norbert Pfeifer, Martin Pfennigbauer, and Gottfried Mandlburger
}

\begin{abstract}
In contrast to topographic laser scanning, laser hydrography must take into account the presence of two media. A pulsed laser beam, which enters the water from the air at an oblique angle, is refracted at the air-water boundary in the direction of the plumb line. This change of direction described by Snellius' law is caused by a slower speed of the light wave in the water, the phase velocity. Light scattering caused by turbidity gives rise to further deviations from the straight path. Together, the slower speed and the turbidity induced path extension cause a longer pulse round trip time in water than in air. For an accurate measurement it is important to correct this propagation time extension. It is common practice to assume the phase velocity as the velocity for the laser pulses in water. In a dispersive medium, however, the phase velocity is only an approximation of the velocity of a pulse. In media with chromatic dispersion, the pulses propagate with a different velocity, the group velocity. In water, using the group velocity instead of the phase velocity reduces the range dependent bias of the depth measurement at a laser wavelength of $532 \mathrm{~nm}$ by more than $1.5 \%$. We present an easy to perform experiment which shows that the group velocity differs so much from the phase velocity that this difference should be taken into account. We further discuss the use of group velocity to explain the depth bias using examples from the literature.
\end{abstract}

Index Terms-Laser hydrography, depth bias, group velocity, chromatic dispersion, refraction correction.

\section{INTRODUCTION}

$\mathbf{L}$ ASER hydrography is an extension of LiDAR for remote sensing of underwater areas and the seabed as well as inland waters such as lakes, rivers and estuaries [1]. While in topographic LiDAR laser pulses only propagate in air, laser hydrography includes water as a second medium. Assuming a simplified model of a flat and horizontal water surface, the direction of the laser beam changes when entering the water. The beam is refracted at the interface of the two media because the speed of light is slower in water than in air. Neglecting the effects of multiple scattering, the laser pulse will eventually hit an object or the ground, from where it is reflected and returns to the sensor via the surface. Under topographic conditions, knowledge of the beam direction and the flight time of the pulse is sufficient to calculate the position of the reflector. In laser hydrography, refraction causes the reflector to appear at a shifted position and must therefore be corrected. Fig. 1 shows the oblique path $r$ under the angle $\vartheta_{w}$ to the object at the point $R$ and its image at the apparent position $S$, the position that would result without correction. In oceanic applications the depth $z$ is of primary importance [2]

$$
z=\frac{T_{w}}{2} v_{w} \cos \left(\vartheta_{w}\right)
$$

Manuscript received September 27, 2020

R. Schwarz, and M. Pfennigbauer are with RIEGL Research Forschungsgesellschaft mbH, 3580 Horn, Austria, e-mail: rschwarz@riegl.com, and mpfennigbauer@riegl.com

N. Pfeifer, and G.Madlburger are with the Department of Geodesy and Geoinformation, TU Wien, Gußhausstr. 27-29, A-1040, Vienna, Austria, e-mail: norbert.pfeifer@geo.tuwien.ac.at, gottfried.mandlburger@geo.tuwien.ac.at

G. Mandlburger is also with the Institute for Photogrammetry, University of Stuttgart, Geschwister-Scholl-Str. 24D, 70174 Stuttgart, Germany, email: gottfried.mandlburger@ifp.unistuttgart.de where $v_{w}$ is the speed of the pulse in water, $\vartheta_{w}$ the angle of refraction and $T_{w}$ the pulse round trip time between surface point $O$ and bottom point $R$. For inland waters a full 3-d correction is applied [3]

$$
\vec{r}=\frac{T_{w}}{2} v_{w} \overrightarrow{k_{w}}
$$

with $\overrightarrow{k_{w}}$ the unit vector of propagation in water. Term $\cos \left(\vartheta_{w}\right)$ and vector $\overrightarrow{k_{w}}$ are determined by Snellius' law,

$$
n_{a} \sin \left(\vartheta_{a}\right)=n_{w} \sin \left(\vartheta_{w}\right)
$$

in scalar

$$
\cos \left(\vartheta_{w}\right)=\sqrt{1-\left(\frac{n_{a}}{n_{w}} \sin \left(\vartheta_{a}\right)\right)^{2}},
$$

or in vector form (see, e.g., [4])

$$
\overrightarrow{k_{w}}=\frac{n_{a}}{n_{w}}\left(\overrightarrow{k_{a}}+\vec{u}\left(\sqrt{\left(\frac{n_{w}}{n_{a}}\right)^{2}-1+\left(\overrightarrow{k_{a}} \cdot \vec{u}\right)^{2}}-\overrightarrow{k_{a}} \cdot \vec{u}\right)\right)
$$

with beam direction vector $\overrightarrow{k_{a}}$ in air, surface normal $\vec{u}, n_{a}$, and $n_{w}$ the refractive indices in air and water, respectively. In both cases, (1) or (2), three factors affect the results: direction, timing, and propagation speed.

The direction vector $\overrightarrow{k_{w}}$ in water depends not only on the surface normal $\vec{u}$ and the beam direction $\overrightarrow{k_{a}}$ in air, but also on the ratio $n_{a} / n_{w}$. The direction of $\vec{u}$ and $\overrightarrow{k_{a}}$ is strongly influenced by surface waves and is discussed in [2], [5], [6]. The refractive indices $n_{a}$ and $n_{w}$ are usually considered constant, at least during a single measurement campaign. A common rule of thumb is to use $n_{w}=4 / 3$ and $n_{a}=1$, although more accurate data is available. Austin and Halikas [7] compiled and tabulated the refractive index of water as a function of salinity, temperature, wavelength and pressure for the visible light spectrum between $400 \mathrm{~nm}$ and $700 \mathrm{~nm}$. Quan and Fry [8] (QAF) calculated an empirical smooth and differentiable function based on these tables. The general uncertainty of incidence angle due to strong surface wave effects, however, does not usually justify the use of the more precise data for the purpose of direction correction.

Timing, the measurement of round trip time $T_{w}$, is the second factor that influences the refraction correction. Accuracy is determined by the properties of the clock oscillator and the uncertainties that arise from the determination of time points in the echo pulses. Typical clock sources such as oven controlled crystal oscillators (OXCO) have a relative accuracy of the oscillation frequency in the order of $10^{-8}$. Consequently, it can be assumed that the decisive factor is not the accuracy of the clock oscillator, but the ability to correctly locate time points from the waveform. Surface uncertainty and propagation-induced pulse-stretching [2] are two sources for timing bias. Guenther et al. [9] proposed a waveform model to predict the bias caused by scattering effects. Other models of the bathymetric echo response waveform e.g. [10]-[12] have been proposed in order to understand and reduce these biases. 


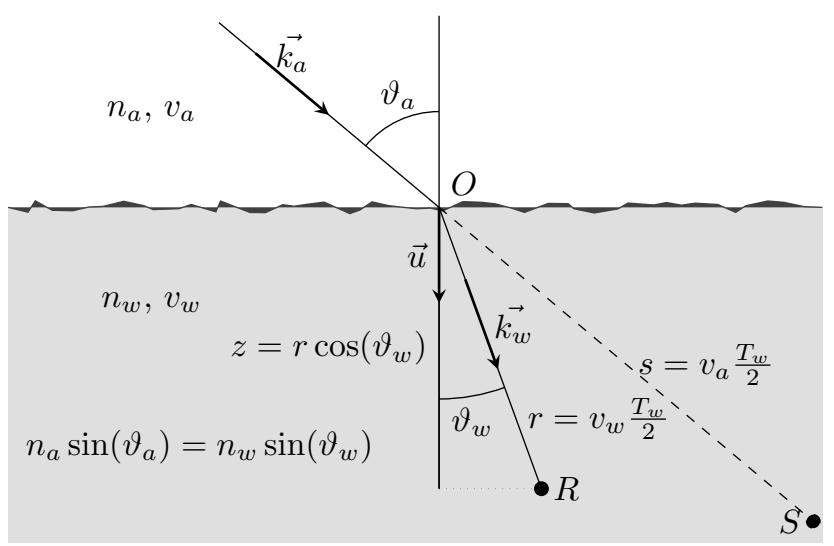

Fig. 1. Refraction at the interface of air and water (proportions are exaggerated). A beam of direction $\overrightarrow{k_{a}}$ entering at nadir angle $\vartheta_{a}$ from air will propagate at direction $\overrightarrow{k_{w}}$, bent towards the surface normal $\vec{u}$ at angle $\vartheta_{w}$. The apparent point $S$ is at slant distance $s$ given by the speed of light in air times half the round trip time $T_{w}$. Position $R$ of the real pulse location is determined by angle $\vartheta_{w}$ and the speed of light in water $v_{w}$. Depth $z$ is obtained as the projection of $r$ to the vertical.

Propagation speed $v_{w}$ of the laser pulse is the third factor in refraction correction. The wave equation predicts that a harmonic light wave will propagate in a medium with speed

$$
v=\frac{c_{0}}{n},
$$

the phase velocity, where $c_{0}$ is the speed of light in vacuo, and $n$ the index of refraction in that medium. For the value of $n$ in water see e.g. [13]. Throughout the literature on laser hydrography, the phase velocity $v$, given by (6), is used as propagation velocity $v_{w}$, which means that $n_{w}$, which is used for the determination of the refraction angle, is also used for the determination of $v_{w}$. In fact a value close to $4 / 3$ is often assumed, e.g. $n_{w}=1.33456$ by Hickman et al. [1], or $n_{w}=1.33182$ by Guenther [2]. Sometimes $n_{w}$ is simply referred to as the refractive index of seawater without specifying a specific value (e.g. Kopilevich et al. [11]), but there is no doubt that the same index is used for direction and speed. The authors of the simulator Wa-LiD [14] use a dedicated symbol for the speed of light in water, but the fact that they simply list $n_{w}=1.33$ as a parameter of the simulator, suggests that they also imply the widespread use of (6) for the speed of the pulses. Similarly, the authors of the articles [15]-[21] assume that the speed in water is given by (6). Westfeld et al. [5] and Yang [18] explicitly point out the dependence of $n_{w}$ on environmental parameters. However, considering QAF formulas with extreme values for $n_{w}$ from 1.334 to 1.343 at $532 \mathrm{~nm}$, the relative depth error should not be able to exceed 1\%. To the best of our knowledge, we found no exception in laser hydrography literature indicating that the light pulses would move at a different speed than that indicated by (6) Therefore, at present in airborne laser bathymetry (ALB) it seems that the same index of refraction is used for direction and range calculation.

The envelope curve of modulated light moves with the group velocity (see e.g. [4], [22]). The simplest modulated waveform $s(x, t)$, described as a function of space $x$ and time $t$, is obtained by superposition of two harmonic waves in a small frequency spacing

$$
s(x, t)=\sin \left(k\left(\omega_{1}\right) x-\omega_{1} t\right)+\sin \left(k\left(\omega_{2}\right) x-\omega_{2} t\right)
$$

where $k(\omega)$ is the angular wave number, i.e., $2 \pi$ times the number of antinodes per length and $\omega_{1}, \omega_{2}$ are the angular frequencies. Using a different set of parameters for the frequencies,

$$
\omega_{1}+\omega_{2}=2 \omega, \text { and } \omega_{1}-\omega_{2}=2 \Delta \omega,
$$

and the approximation

$$
k(\omega \pm \Delta \omega)=k(\omega) \pm \frac{\mathrm{d} k(\omega)}{\mathrm{d} \omega} \Delta \omega,
$$

which is valid for a narrow bandwith, the modulated wave can be factorized as

$$
s(x, t)=2 \sin \left(\omega\left[x \frac{k(\omega)}{\omega}-t\right]\right) \cos \left(\Delta \omega\left[x \frac{\mathrm{d} k(\omega)}{\mathrm{d} \omega}-t\right]\right)
$$

The first factor, the carrier, oscillates with $\omega$ and the second factor, the modulation envelope, oscillates with the considerably lower frequency $\Delta \omega$. The fractions within the square brackets have the dimension of the reciprocal of speed

$$
\frac{1}{v}=\frac{k(\omega)}{\omega}, \quad \text { and } \quad \frac{1}{v_{g}}=\frac{\mathrm{d} k(\omega)}{\mathrm{d} \omega},
$$

the phase velocity $v$ which is the velocity of the carrier, already defined by (6), and the group velocity $v_{g}$ which can be identified as velocity of the envelope.

For harmonic waves to be valid as a solution to Maxwell's equations, they must satisfy the dispersion relation

$$
k(\omega)=\omega \frac{n(\omega)}{c_{0}}
$$

which establishes a relationship between wave number and frequency in a medium, which can be seen to be equivalent to (6). Similar to the refractive index $n$, which is a property of the medium, a group index $n_{g}$ can be defined as

$$
v_{g}=\frac{c_{0}}{n_{g}} .
$$

Rearranging (13), using (11), and (12) the group index can be written

$$
n_{g}(\omega)=n(\omega)+\omega \frac{\mathrm{d} n(\omega)}{\mathrm{d} \omega} .
$$

Usually the refractive index is known as a function of free space wavelength $\lambda$, defined by the free space dispersion relation $\lambda \nu=c_{0}$ rather than frequency $\omega=2 \pi \nu$. Using $\lambda$ instead of $\omega$ equation (14) can be rewritten in the more common form

$$
n_{g}(\lambda)=n(\lambda)-\lambda \frac{\mathrm{d} n(\lambda)}{\mathrm{d} \lambda}
$$

Decisive for how much the group velocity differs from the phase velocity is the rate of change of the refractive index as a function of the frequency or the free space wavelength within the bandwidth of the laser source and the pulse. It is important to note that this result does not depend on the exact shape of the pulse, but only on the narrow-band nature of the process, i.e. the validity of (9).

Since no sufficiently depth bias free models exist, several empirical correction models have been developed [17], [20], [23], [24]. In the following sections we will show that the difference between group and phase velocities in water is large enough that a significant part of the depth bias can be removed by applying physical principles.

\section{A. Measurement of Pulse Delay}

\section{Methods}

A two phase differential scheme was chosen for the measurement of propagation delay. First a measurement chamber is filled with air then with water. The total time for a laser pulse between source and sensor for the two phases are sums

$$
T_{a}=\sum_{i} T_{i}+\tau_{a} \quad \text { and } \quad T_{w}=\sum_{i} T_{i}+\tau_{w}
$$




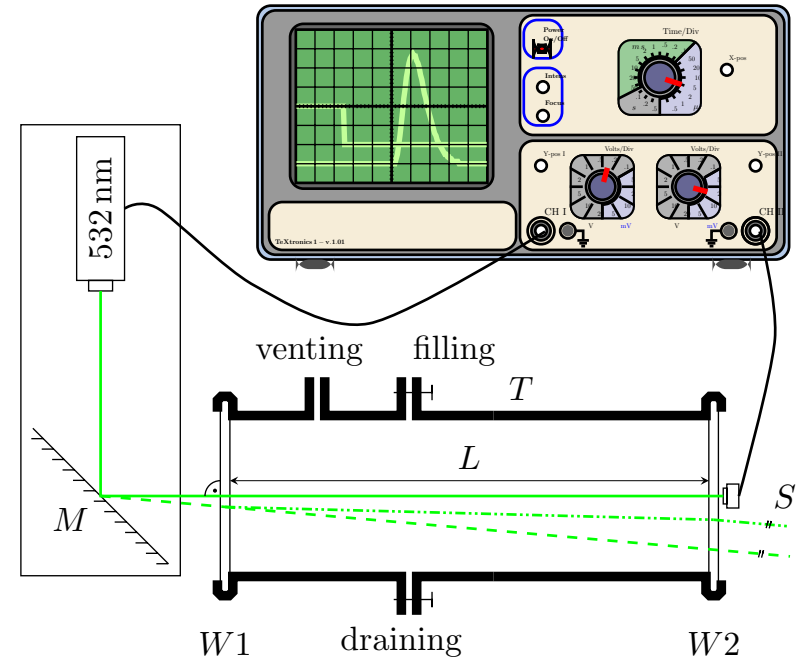

Fig. 2. Experimental setup: Tube $T$ of known length $L$ can be filled with air or water. When the path of the laser pulse (solid green line) is aligned perpendicularly to window $W 1$ by mirror $M$, sensor $S$ can remain at a fixed position. The delay of the pulse is measured between a trigger signal from the laser unit and the sensor $S$. The difference of delays between filled and empty states of $T$ reveals the delay caused by water only.

of the delay $\tau_{w}$ caused by the part of the path containg water, or the delay $\tau_{a}$ of the same part without the water during the other phase, and delays $T_{i}$ caused by the various remaining components of the setup. Since the parts causing delays $T_{i}$ are unaltered between the two phases of the experiment, $T_{a}$ and $T_{w}$ will change only because $\tau_{a}$ and $\tau_{w}$ are different. The difference therefore is equal to the delay difference

$$
\tau_{w}-\tau_{a}=T_{w}-T_{a}
$$

of water and air paths under consideration. Further, because

$$
\tau_{a}=\frac{L}{v_{g}^{a}}=L \frac{n_{g}^{a}}{c_{0}} \quad \text { and } \quad \tau_{w}=\frac{L}{v_{g}^{w}}=L \frac{n_{g}^{w}}{c_{0}}
$$

with $L$ the probe length and $v_{g}^{a}, v_{g}^{w}$ the group velocities in air and water, respectively, the following relation for the indices of group refraction $n_{g}^{a}$ and $n_{g}^{w}$ can be established

$$
n_{g}^{w}=\frac{T_{w}-T_{a}}{L} c_{0}+n_{g}^{a}
$$

The experimental setup is shown in Fig. 2. A Q-switched MicroChip NanoLaser with $532 \mathrm{~nm}$ emission wavelength and a symmetric pulse of $1 \mathrm{~ns}$ width (FWHM) was used as source. The tube $T$ can be filled with tap water, it has nozzles for filling, emptying and pressure equalization. Both ends of the tube are closed by two glass windows $2 \mathrm{~mm} W 1$ and $W 2$. For the distance $L$ between the windows the value $L=986.3 \mathrm{~mm}$ was found by measuring. The direction of the laser is adjusted by means of the mirror $M$ so that it enters $W 1$ perpendicularly, since under these conditions the path is not deflected by refraction in case the tube is filled with water. The effect of a non-perpendicular entry can be seen in Fig. 2 when comparing the refracted (dashed-dotted) beam with the un-refracted (dashed) beam leaving the second window $W 2$. They would not exit the tube $T$ at the same point.

The time intervals $T_{w}$ and $T_{a}$ of the equation 17 were determined with the help of a sampling oscilloscope DPO 1504 manufactured by company Tektronics, bandwidth $1 \mathrm{GHz}$, a Si-PIN photo sensor $S$ Hamamatsu S5973-02 with an active area of $0.4 \mathrm{~mm}$ and corresponding post-processing. The signals detected by the sensor were

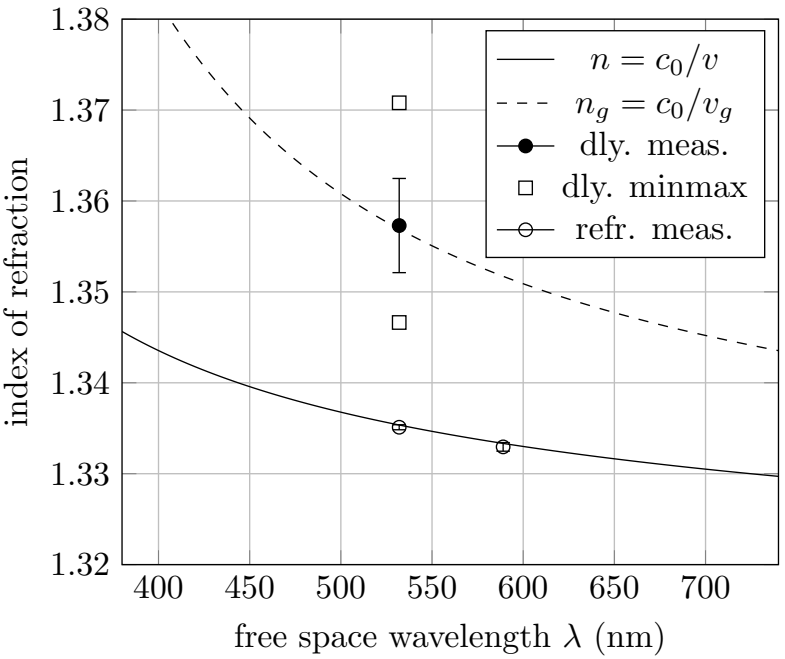

Fig. 3. Results of experiment: The filled circle (dly. meas.) is at the mean value of the group index of the delay measurement, bars delimit the one sigma standard uncertainity $\left(532 \mathrm{~nm}: n_{g}=1.357 \pm 0.005\right) ; \mathrm{min} / \mathrm{max}$ measured values (dly. minmax) 1.347 for the minimum and 1.371 for the maximum, indicated by boxes, correspond to a 79 ps delay spread; Abbé refractometer measurement results (refr. meas.) at $532 \mathrm{~nm}$ are $n=1.3351 \pm 0.0003$ and at $589 \mathrm{~nm} n=1.3329 \pm 0.0005$ ); reference data (solid line) is the formula of [8] for the index of refraction of water $n(\lambda)=c_{0} / v(\lambda)$ (salinity $=0.01 \%$, temperature $=16.4{ }^{\circ} \mathrm{C}$ ); group index $n_{g}(\lambda)=c_{0} / v_{g}(\lambda)$ (dashed line) is calculated from $n$.

recorded, triggered by an electrical pulse synchronized to the emission time of the laser pulse. The oscilloscope settings were the same for measurements with air and water to avoid errors due to different gain settings. The center of gravity was extracted from the recorded waveforms and used to determine the delays.

\section{B. Measurement of the Index of Refraction}

The refractive index, which determines the change in beam direction, was determined with an Abbé laboratory refractometer, which is based on the principle of the critical angle of total reflection. Two series of ten measurements were performed, each for the wavelengths $589 \mathrm{~nm}$ and $532 \mathrm{~nm}$. The first wavelength is the standard wavelength of the instrument. The second wavelength was used to match the pulse delay measurements. All wavelengths were taken from the respective manufacturer's specifications. Different light sources were used for pulse and Abbé measurements.

\section{Calculation of the Group Index}

QAF [8] published an empirical formula of the refractive index of water $n$ as a function of salinity, temperature, and wavelength. By using (15) and calculating the derivative of $n$ an explicit formula for $n_{g}$ was obtained. Fig. 3 shows the resulting curve of the group index as a dashed line.

\section{RESUlts AND Discussion}

\section{A. Experimental Determination of Group Velocity in Water}

The result of the experiment shows that group velocity and phase velocity indeed differ by $1.8 \%$ at $\lambda=532 \mathrm{~nm}$ in water. Table I lists results of the delay measurement relevant for group velocity and Table II lists results of refraction measurement relevant for phase velocity. Five water filling and emptying cycles of the pipe were performed. Each line of the table I showing a cycle consists of two successive measurements in water, $T_{w}^{1}, T_{w}^{2}$, followed by two successive 
TABLE I
DELAYS $T_{w}^{1,2}$ IN WATER AND $T_{a}^{1,2}$ IN AIR NANO SECONDS. FIVE CYCLES OF FILLING AND DRAINING, TWO MEASUREMENTS PER CYCLE.

\begin{tabular}{ccccc}
\hline Cycle & $T_{w}^{1}$ & $T_{w}^{2}$ & $T_{a}^{1}$ & $T_{a}^{2}$ \\
\hline 1 & 4.770 & 4.770 & 3.590 & 3.584 \\
2 & 4.784 & 4.776 & 3.598 & 3.603 \\
3 & 4.772 & 4.764 & 3.606 & 3.618 \\
4 & 4.762 & 4.762 & 3.573 & 3.565 \\
5 & 4.758 & 4.761 & 3.594 & 3.603
\end{tabular}

TABLE II

REFRACTION INDICES FROM ABBÉ REFRACTOR MEASUREMENTS.

\begin{tabular}{cccccc}
\hline$\lambda /$ meas. & 1 & 2 & 3 & 4 & 5 \\
\hline $532 \mathrm{~nm}$ & 1.3359 & 1.3350 & 1.3350 & 1.3349 & 1.3351 \\
$589 \mathrm{~nm}$ & 1.3324 & 1.3332 & 1.3332 & 1.3325 & 1.3340 \\
\hline$\lambda /$ meas. & 6 & 7 & 8 & 9 & 10 \\
\hline $532 \mathrm{~nm}$ & 1.3350 & 1.3351 & 1.3350 & 1.3349 & 1.3352 \\
$589 \mathrm{~nm}$ & 1.3334 & 1.3328 & 1.3327 & 1.3328 & 1.3325 \\
\hline
\end{tabular}

measurements in air $T_{a}^{1}, T_{a}^{2}$. The delay measurements were converted into a group refraction index for water by (19) using the length of the water probe $L=986.3 \mathrm{~mm}$ and the group refraction index in air $n_{g}^{a}=1.000281$ which was determined from the environmental conditions. The black dot in Fig. 3 is the mean value of the deceleration measurements, which gives $n_{g}^{w}=1.357$ with 0.005 standard deviation. The extreme values are 1.371 for the maximum and 1.347 for the minimum.

Table II lists two rows of refraction indices one for the wavelength $\lambda=532 \mathrm{~nm}$ that has been used in the delay experiment and a second $\lambda=589 \mathrm{~nm}$ the sodium $D$ line. The mean values of both refraction measurements are shown in Fig. 3 as two circles at $n=1.3351$ with standard deviation $3 \times 10^{-4}$ for $\lambda=532 \mathrm{~nm}$ and $n=1.3329$ with standard deviation $5 \times 10^{-4}$ for $\lambda=589 \mathrm{~nm}$.

All measurements show good agreement with the results obtained from the QAF formulae.

\section{B. Interpretation of Depth Bias in Context of Group Velocity}

A few examples from the literature allow a direct interpretation as an effect of chromatic dispersion. Especially for errors that are proportional to depth, in clear waters and great depths, beyond $2 \mathrm{~m}$, chromatic dispersion offers a good explanation.

Wright et al. [17] conducted a depth calibration of the EAARL-B sensor. They found a linear dependence on depth which could be corrected by using a simple linear regression analysis. Their correction formula reads

$$
y=0.98103 D_{e b}-0.00068
$$

where $D_{e b}$ is the uncorrected EAARL-B depth measurement and $y$ is the corrected depth. The mean difference (the error) $y-D_{e b}$ is plotted as a dash-dotted line in Fig. 4. The authors of [17] report on the use of $n=1.333$ for the value of the refractive index. Assuming that the same value was used to convert between time and range, the time delay $t$ can be reconstructed by

$$
t=\frac{2 \cdot 1.333}{c_{0}} D_{e b}
$$

Assuming that the laser pulses propagate at group velocity, the depths can be calculated as follows

$$
y_{g}=\frac{v_{g}}{2} t=\frac{c_{0}}{2 n_{g}} t=\frac{1.333}{n_{g}} D_{e b} \text {. }
$$

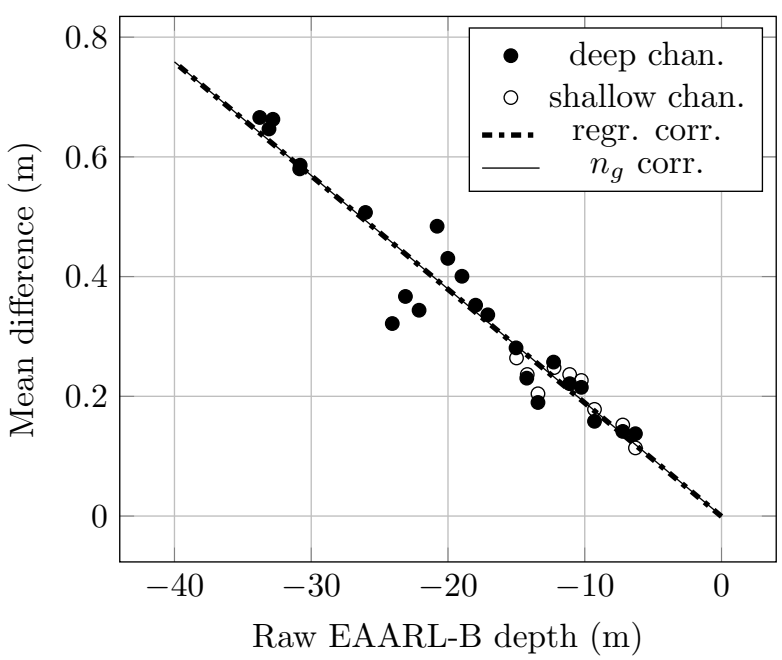

Fig. 4. Scatterplot data taken from sensor calibration by [17] demonstrating, that the observed depth differences could be explained by application of a group index $n_{g}=1.359$ (salinity $=1.25 \%$, temperature $=24^{\circ} \mathrm{C}$ ) instead of $n=1.333$ which was used in the article.

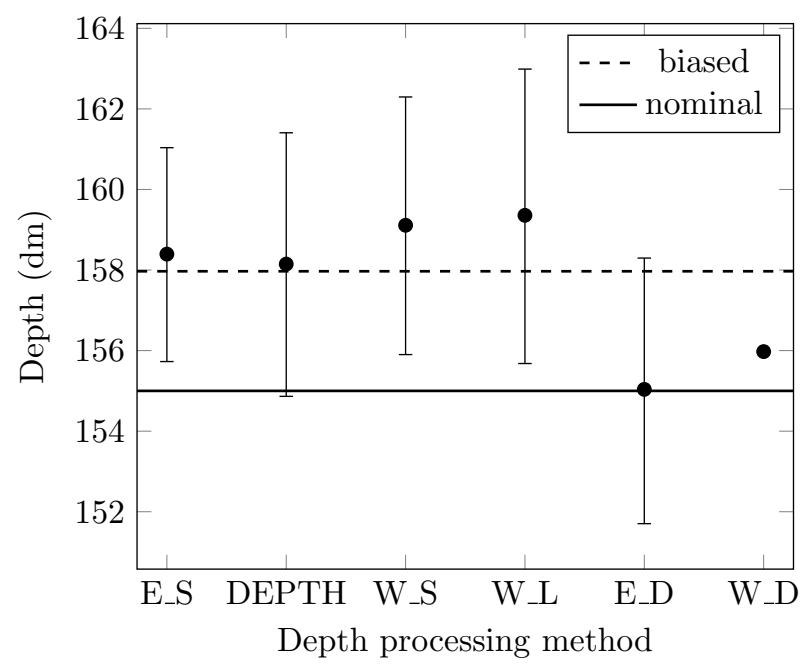

Fig. 5. Error bar plot taken from experimental evaluation by [25]. The black line is at the nominal (true) depth. The dashed line shows chromatic dispersion bias for $\lambda=532 \mathrm{~nm}$, salinity $=0.7 \%$, temperature $\approx 9.5^{\circ} \mathrm{C}$, and a value of $n=4 / 3$. Horizontal axis labels name various depth extraction algorithms, see [25] for details.

A specific choice ${ }^{1}$ of the water temperature and the use of the QAF formulas shows that it is possible to make the depth bias disappear by using the group velocity for the velocity of the laser pulses. The solid line $y_{g}-D_{e b}$ and the line found by linear regression are practically indistinguishable. The value of the diffuse attenuation coefficient was given in the article as $K=0.1 \mathrm{~m}^{-1}$, which indicates clear water.

Steinvall et al. [25] reported a dept bias for which they had no explanation, although they had made a correction to the depth bias as suggested by Guenther [2]. Fig. 5 reproduces a graph from their depth accuracy report. Stated water parameters were $K=0.22 \mathrm{~m}^{-1}$, salinity $=0.7 \%$, and temperature $\approx 9.5^{\circ} \mathrm{C}$. Using those numbers, the bias from having used $n=4 / 3$ instead of $n_{g}=1.3589$ is

$$
155.0 \mathrm{dm} \frac{1.3589}{4 / 3}=158.0 \mathrm{dm} \text {, }
$$

\footnotetext{
${ }^{1}$ Temperature and salinity recordings were not available from the article.
} 
which has been added to Fig. 5 as dashed line. A later publication [26] of the same authors contains a comparison of laser and acoustic data stating the relationship of

$$
\text { Acoustic depth }=0.9804 \times \text { Laser depth }-0.1745
$$

describing a $2 \%$ depth bias which could also be explained by chromatic dispersion.

The results of our own research [21] show, however, that further investigations will be necessary to fully understand the depth bias also in turbid waters and shallower depths. A recent study [27] also shows that for turbid waters the explanation by chromatic dispersion alone is obviously not sufficient.

Not unexpectedly, it turns out that the consideration of chromatic dispersion alone cannot explain the full extent of depth bias. We suspect that for higher turbidity levels, multiple scattering causes additional error. However, we are convinced that existing algorithms should be interpreted in the context of the slower velocity. In any case, we have experimentally confirmed that the group velocity differs so much from the phase velocity in the medium water that this difference must be taken into account. The result of the experiment is in line with the interpretation of the physical literature applied to laser hydrography.

\section{CONCLUSION}

We have shown that the effect of chromatic dispersion in water shall not be neglected for laser hydrography. A consequence of chromatic dispersion is a propagation speed slower than phase velocity. This group velocity, is $1.8 \%$ smaller at a wavelength of $532 \mathrm{~nm}$. We therefore propose that $n$ be replaced by $n_{g}$ for the calculation of the velocity of propagation in pulsed laser hydrography. This corrects the scale for the conversion between time and length and reduces the depth bias. The value of $n_{g}$ can be calculated from (15) using tabular data of $n(\lambda)$ or from an empirical formula given by [8].

Routine recording of salinity and temperature of the water would be advantageous for the purposes of refraction correction in postprocessing. However, a default value of $n_{g}=1.36$ can be used as a compromise, instead of the usual $4 / 3$, when no measurement data is available.

\section{ACKNOWLEDGMENT}

The authors express their gratitude to company Riegl for the supply of materials and instruments used for the experiment. The work of R.S. and M.P. was funded by Austrian Research Funding Agency (FFG) within the project "Aerial search \& Rescue support and supErvision of inAccessible terrainS" (AREAS) 86702, the work of G.M. was funded by German Research Foundation (DFG) for project SO 935/6-1, N.P. received no external funding for this research.

\section{REFERENCES}

[1] G. D. Hickman and J. E. Hogg, "Application of an airborne pulsed laser for near shore bathymetric measurements," Remote Sensing of Environment, vol. 1, no. 1, pp. 47-58, 1969.

[2] G. C. Guenther, Airborne laser hydrography system design and performance factors, ser. NOAA Professional Paper Series, N. O. S. 1, Ed. National Oceanic and Atmospheric Administration, Rockville, MD,20852, 1985

[3] M. Pfennigbauer, A. Ullrich, F. Steinbacher, and M. Aufleger, "Highresolution hydrographic airborne laser scanner for surveying inland waters and shallow coastal zones," in Laser Radar Technology and Applications XVI, M. D. Turner and G. W. Kamerman, Eds. SPIE, 52011.

[4] E. Hecht, Optics, 5th Edition. Pearson, 2016.

[5] P. Westfeld, H.-G. Maas, K. Richter, and R. Weiß, "Analysis and correction of ocean wave pattern induced systematic coordinate errors in airborne LiDAR bathymetry," ISPRS Journal of Photogrammetry and Remote Sensing, vol. 128, pp. 314-325, 62017.
[6] K. Richter, D. Mader, P. Westfeld, and H.-G. Maas, "Numerical simulation and experimental validation of wave pattern induced coordinate errors in airborne lidar bathymetry," ISPRS - International Archives of the Photogrammetry, Remote Sensing and Spatial Information Sciences, vol. XLII-2, pp. 961-967, 52018.

[7] R. W. Austin and G. Halikas, "The index of refraction of seawater," Scripps Institution of Oceanography La Jolla Ca Visibility Lab, Tech. Rep., 1976.

[8] X. Quan and E. S. Fry, "Empirical equation for the index of refraction of seawater," Applied Optics, vol. 34, no. 18, p. 3477, 61995.

[9] G. C. Guenther and R. W. Thomas, "Effects of propagation-induced pulse stretching in airborne laser hydrography," in Ocean Optics VII M. A. Blizard, Ed. SPIE, 71984.

[10] R. E. Walker and J. W. McLean, "Lidar equations for turbid media with pulse stretching," Applied Optics, vol. 38, no. 12, p. 2384, 41999

[11] Y. I. Kopilevich and A. G. Surkov, "Mathematical modeling of the input signals of oceanological lidars," Journal of Optical Technology, vol. 75, no. 5 , p. 321,52008 .

[12] R. Schwarz, N. Pfeifer, M. Pfennigbauer, and A. Ullrich, "Exponentia decomposition with implicit deconvolution of lidar backscatter from the water column," PFG - Journal of Photogrammetry, Remote Sensing and Geoinformation Science, vol. 85, no. 3, pp. 159-167, 72017.

[13] C. D. Mobley, Light and water: radiative transfer in natural waters. Academic Press, 1994.

[14] H. Abdallah, N. Baghdadi, J.-S. Bailly, Y. Pastol, and F. Fabre, "Wa-LiD A new LiDAR simulator for waters," IEEE Geoscience and Remote Sensing Letters, vol. 9, no. 4, pp. 744-748, 2012.

[15] M. F. Penny, R. H. Abbot, D. M. Phillips, B. Billard, D. Rees, D. W. Faulkner, D. G. Cartwright, B. Woodcock, G. J. Perry, P. J. Wilsen, T. R. Adams, and J. Richards, "Airborne laser hydrography in australia," Applied Optics, vol. 25, no. 13, pp. 2046-2058, 71986.

[16] A. Bouhdaoui, J.-S. Bailly, N. Baghdadi, and L. Abady, "Modeling the water bottom geometry effect on peak time shifting in LiDAR bathymetric waveforms," IEEE Geoscience and Remote Sensing Letters, vol. 11, no. 7, pp. 1285-1289, 72014.

[17] C. W. Wright, C. Kranenburg, T. A. Battista, and C. Parrish, "Depth calibration and validation of the experimental advanced airborne research lidar, EAARL-b," Journal of Coastal Research, vol. 76, pp. 4-17, 12 2016

[18] F. Yang, D. Su, Y. Ma, C. Feng, A. Yang, and M. Wang, "Refraction correction of airborne LiDAR bathymetry based on sea surface profile and ray tracing," IEEE Transactions on Geoscience and Remote Sensing, vol. 55, no. 11, pp. 6141-6149, 112017.

[19] S. Xing, D. Wang, Q. Xu, Y. Lin, P. Li, L. Jiao, X. Zhang, and C. Liu, "A depth-adaptive waveform decomposition method for airborne lidar bathymetry," Sensors, 2019.

[20] C. E. Parrish, L. A. Magruder, A. L. Neuenschwander, N. ForfinskiSarkozi, M. Alonzo, and M. Jasinski, "Validation of ICESat-2 ATLAS bathymetry and analysis of ATLAS's bathymetric mapping performance,' Remote Sensing, vol. 11, no. 14, p. 1634, 72019.

[21] R. Schwarz, G. Mandlburger, M. Pfennigbauer, and N. Pfeifer, "Design and evaluation of a full-wave surface and bottom-detection algorithm for LiDAR bathymetry of very shallow waters," ISPRS Journal of Photogrammetry and Remote Sensing, vol. 150, pp. 1-10, 42019.

[22] S. J. Orfanidis, Electromagnetic Waves and Antennas. Sophocles J. Orfanidis, 2016. [Online]. Available: http://eceweb1.rutgers.edu/ $\sim$ orfanidi/ewa/ewa- lup.pdf

[23] B. Billard, R. H. Abbot, and M. F. Penny, "Modeling depth bias in an airborne laser hydrographic system," Applied Optics, vol. 25, no. 13, p. 2089, 71986

[24] J. Zhao, X. Zhao, H. Zhang, and F. Zhou, "Improved model for depth bias correction in airborne LiDAR bathymetry systems," Remote Sensing, vol. 9, no. 7, p. 710, 2017. [Online]. Available: http://www.mdpi.com/2072-4292/9/7/710

[25] O. K. Steinvall, K. R. Koppari, and U. C. M. Karlsson, "Experimental evaluation of an airborne depth-sounding lidar," Optical Engineering, vol. 32 , no. 6, p. $1307,1993$.

[26] _ - "Airborne laser depth sounding: system aspects and performance," in Ocean Optics XII, J. S. Jaffe, Ed. SPIE, 101994

[27] G. Mandlburger, M. Pfennigbauer, R. Schwarz, S. Flöry, and L. Nussbaumer, "Concept and performance evaluation of a novel UAV-borne topo-bathymetric LiDAR sensor," Remote Sensing, vol. 12, no. 6, p. 986 , mar 2020 . 